THE TRANSVERSALITY RELATIVE TO A SURFACE OF $\int F\left(x, y, z, y^{\prime}, z^{\prime}\right) d x=$ MINIMUM* BY JESSE DOUGLAS $\dagger$

1. Introduction and Statement of Theorem. Consider, for illustration, a surface $\Sigma$, on which $A$ and $B$ are any two points. Then, of all curves in space which join $A$ and $B$, the straight line segment $A B$ is the shortest; of all curves on $\Sigma$ joining $A$ and $B$, the geodesic $A B$ is the shortest.

The generalization from the length integral

$$
s=\int\left(1+y^{\prime 2}+z^{\prime 2}\right)^{1 / 2} d x
$$

to the general integral of first order

$$
J=\int F\left(x, y, z, y^{\prime}, z^{\prime}\right) d x
$$

is obvious. Thus we may speak on the one hand of the unrestricted extremals of $J$ relative to space, $\infty^{4}$ in number, and, on the other, of the extremals of $J$ relative to a given surface $\Sigma, \infty^{2}$ in number.

The idea of transversality may likewise be defined relative to a given surface as well as for space. Let us review the wellknown definitions and facts in this connection. The space transversality $T$ belonging to an integral $J$ is essentially a correspondence between lineal elements and surface elements (of the first order) characterized by the following two properties: (1) a lineal element and its corresponding surface element have the same base point, (2) if, taking an arbitrary base surface $S$, we construct the $\infty^{2}$ extremals of $J$ which meet $S$ transversally, then lay off along each extremal, starting at $S$, an arc over which the integral $J$ has a fixed value, the locus of the end points of these arcs is a surface trans-

* Presented to the Society, October 30, 1926.

$\dagger$ National Research Fellow in Mathematics. 
versal to the congruence of extremals. By varying the fixed value continuously $\infty^{1}$ such transversal surfaces result.

Analytically, if a lineal element be fixed by coordinates $x, y, z, y^{\prime}, z^{\prime}$ in the usual way, the transverse surface element has the same base point $x, y, z$, and the equation of its (infinitesimal piece of a) plane is

$$
\left(F-y^{\prime} F_{y^{\prime}}-z^{\prime} F_{z^{\prime}}\right) \delta x+F_{y^{\prime}} \delta y+F_{z^{\prime}} \delta z=0,
$$

where $\delta x, \delta y, \delta z$ denote coordinates relative to $x, y, z$.

The surface $S$ or any of the other transversal surfaces of the congruence of extremals may degenerate into a curve or a point-in fact, the appropriate concept to use here is not that of surface but Lie's concept of union of surface elements. The simplest representation of a transversality is obtained by allowing $S$ to be a point-the extremals transversal to a point are simply those which issue from it. In the illustrative case of the length integral (1) the transversal surfaces are then concentric spheres, from which it appears that for the length integral transversality is the same as orthogonality* - a fact which is expressed analytically by the circumstance that the equation (3) formed for (1) reduces to $\delta x+y^{\prime} \delta y+z^{\prime} \delta z=0$.

How is the transversality of $J$ relative to an arbitrary surface $\Sigma$ defined? Let us construct the pencil of extremals of $J$ relative to $\Sigma$ which radiate from a fixed point 0 of $\Sigma$. If we proceed along each extremal, starting from 0 , until we have run off a fixed value of $J$, the locus of points arrived at is a curve $\Gamma$, which we say meets transversally the extremals radiating from 0 . More definitely, if $C$ is one of the extremals through 0 , intersecting $\Gamma$ at point $p$, the lineal element $\lambda$ of $\Gamma$ at $p$ is termed transversal to the lineal element $l$ of $C$ at the same point. By varying 0 , there is assigned to each lineal element $l$ of $\Sigma$ a transverse lineal element $\lambda$.

* The most general integral of the first order for which transversality is orthogonality is $\int \nu(x, y, z) d s$, where $\nu$ is any function of its three arguments. 
If $C$ is the extremal containing the element $l$, any point of $C$ may be used as point 0 to define the transverse element $\lambda$; we always get the same $\lambda$.

The correspondence so defined between $l$ and $\lambda$ we call the transversality of $J$ relative to $\Sigma$, and denote by $\tau$; it is of course to be carefully distinguished from the space transversality $T$ of $J$. The analogy between $\tau$ and $T$ is completed by the theorem that the extremals of $J$ relative to $\Sigma$ which start transversally from any base curve have $\infty^{1}$ transversal curves, between any two of which arcs over which $J$ has the same value are intercepted on all these extremals.

Now, in the theory of surfaces there is a classic theorem of Gauss which may be stated as follows.

Any two orthogonal trajectories of a system of $\infty^{1}$ geodesics of a surface intercept the same length on all these geodesics.*

In our terminology the theorem may be stated as follows:

The transversality $\tau$ of the length integral relative to an arbitrary surface $\Sigma$ is the orthogonality of lineal elements on $\Sigma$.

The purpose of the present paper is to prove the following more general theorem, which includes that of Gauss as a special case.

THEOREM. The transversality $\tau$ of any integral $J$ relative to an arbitrary surface $\Sigma$ is the section by $\Sigma$ of the space transversality $T$ of $J$.

By the section of $T$ by $\Sigma$ we mean, naturally, the following: Let $l$ be any lineal element of $\Sigma$, and let the surface element $\sigma$ correspond to $l$ by $T$; then the lineal element $\lambda$ corresponding to $l$ by $\tau$ is the intersection of (the tangent plane of) $\Sigma$ with $\sigma$.

2. Proof. Let $\Sigma$ be represented by the equation

$$
x+\phi(y, z)=0 .
$$

* G. Scheffers, Einführung in die Theorie der Flächen, second edition, 1913, p. 500. 
Let

$$
y=y(x), \quad z=z(x)
$$

define a curve $C$ on $\Sigma$ which joins any two fixed points 0 and 1 of that surface. Then the infinitely near curve $\bar{C}$

$$
y=y(x)+\epsilon \eta(x), \quad z=z(x)+\epsilon \zeta(x)
$$

may be considered as lying on $\Sigma$ when and only when

$$
p \eta(x)+q \zeta(x)=0
$$

where $p=\phi_{y}(y, z), q=\phi_{z}(y, z)$ and $y, z$ are to be replaced by the functions of $x$ appearing in (5); (7) then to hold identically in $x$.

The conditions that $\bar{C}$ pass through 0 and 1 are

$$
\eta\left(x_{0}\right)=0, \zeta\left(x_{0}\right)=0 ; \eta\left(x_{1}\right)=0, \zeta\left(x_{1}\right)=0 .
$$

Supposing (7) and (8) verified, the variation of $J$ in passing from $C$ to $\bar{C}$ is, by means of the usual integration by parts,

$$
\delta J=\int_{0}^{1}\{A(F) \eta+B(F) \zeta\} d x
$$

where

$$
A(F)=F_{y}-\frac{d}{d x} F_{y^{\prime}}, B(F)=F_{z}-\frac{d}{d x} F_{z^{\prime}},
$$

$d / d x$ denoting total differentiation with respect to the abscissa of $C$. In order that $C$ be an extermal of $J$ relative to $\Sigma$, the necessary and sufficient condition is

$$
A(F) \eta+B(F) \zeta=0
$$

for all $\eta, \zeta$ satisfying (7), or $q A(F)-p B(F)=0$ where all the symbols involved are to be reduced to functions of $x$, abscissa along $C$.

Suppose now 2 to denote any point on $\Sigma$ infinitely near to 1 , and let $C^{\prime}$ be the extremal relative to $\Sigma$ which joins 0 to 2 . Its equations have the form (6) with (7) verified, and $\eta\left(x_{0}\right)=0, \zeta\left(x_{0}\right)=0$, but instead of the second pair of equations (8) we have, expressive of the fact that $C^{\prime}$ contains 2 , 


$$
\delta y=y_{1}^{\prime} \delta x+\epsilon \eta\left(x_{1}\right), \delta z=z_{1}^{\prime} \delta x+\epsilon \zeta\left(x_{1}\right),
$$

where $\delta x, \delta y, \delta z$ denote the coordinates of 2 relative to 1 and $x_{1}, y_{1}, z_{1}, y_{1}^{\prime}, z_{1}^{\prime}$ are the coordinates of the lineal element of $C$ at 1 .

These equations then follow:

$$
\begin{aligned}
J_{01}= & \int_{x_{0}}^{x_{1}} F\left(x, y, z, y^{\prime}, z^{\prime}\right) d x, \\
J_{02}= & \int_{x_{0}}^{x_{2}+\delta x}\left\{F+\epsilon\left(F_{y} \eta+F_{z} \zeta+F_{y^{\prime}} \eta^{\prime}+F_{z^{\prime}} \zeta^{\prime}\right)\right\} d x \\
= & \int_{x_{0}}^{x_{1}}\left\{F+\epsilon\left(F_{y} \eta+F_{z} \zeta+F_{y^{\prime}} \eta^{\prime}+F_{z^{\prime}} \zeta^{\prime}\right)\right\} d x \\
& +F\left(x_{1}, y_{1}, z_{1}, y_{1}^{\prime}, z_{1}^{\prime}\right) \delta x,
\end{aligned}
$$

where we neglect infinitesimals of higher than the first order. By subtraction we find

$$
\begin{aligned}
\delta J= & J_{02}-J_{01} \\
=\epsilon & \int_{x_{0}}^{x_{1}}\left(F_{y} \eta+F_{z} \zeta+F_{y^{\prime}} \eta^{\prime}+F_{z^{\prime}} \zeta^{\prime}\right) d x+F\left(x_{1}, y_{1}, z_{1}, y_{1}^{\prime}, z_{1}^{\prime}\right) \delta x \\
= & \epsilon\left\{F_{y^{\prime}}(1) \eta\left(x_{1}\right)+F_{z^{\prime}}(1) \zeta\left(x_{1}\right)\right\} \\
& \quad+\epsilon \int_{x_{0}}^{x_{1}}\{A(F) \eta+B(F) \zeta\} d x+F(1) \delta x .
\end{aligned}
$$

The integral vanishes by (11), since $\eta, \zeta$ satisfy ( 7 ) because $C^{\prime}$ lies on $\Sigma$. Using (12), we have after dropping the index 1,

$$
\delta J=\left(F-y^{\prime} F_{y^{\prime}}-z^{\prime} F_{z^{\prime}}\right) \delta x+F_{y^{\prime}} \delta y+F_{z^{\prime}} \delta z .
$$

For transversality, $\delta J=0$, giving

$$
\left(F-y^{\prime} F_{y^{\prime}}-z^{\prime} F_{z^{\prime}}\right) \delta x+F_{y^{\prime}} \delta y+F_{z^{\prime}} \delta z=0 .
$$

Besides (14), $\delta x, \delta y, \delta z$ are restricted to satisfy

$$
\delta x+p \delta y+q \delta z=0,
$$

which is the equation of the element of $\Sigma$ in the immediate neighborhood of 1 (tangent plane). The direction $\delta x: \delta y: \delta z$ transverse to $1: y^{\prime}: z^{\prime}$ at 1 is to be found by combining (14) with (15). 
Now (14) is identical with (3) defining the space transversality of $J$. Hence our theorem.

The theorem is easily extended to an $m$ space immersed in an $n$ space. Since a Riemann space of $m$ dimensions can always be immersed in a flat space of at most $\frac{1}{2} m(m+1)$ dimensions, we have an immediate proof of the Gauss theorem for a Riemann space of any number of dimensions. For it is obvious that the transversality of the length integral in a flat $n$-space is the orthogonality of lineal elements to $(n-1)$-elements with the same base point, and evidently the section of this transversality by any $m$-spread contained in the $n$-flat is the orthogonality of lineal to $(m-1)$-elements in the $m$-spread.

Princeton University

\section{ON THE EXTENSION OF A METHOD OF BRIOT AND BOUQUET FOR THE REDUCTION OF SINGULAR POINTS*}

BY B. O. KOOPMAN

In a classical memoir, $\dagger$ Briot and Bouquet gave a method by means of which the differential equation

$$
\frac{d x}{X(x, y)}=\frac{d y}{Y(x, y)}
$$

could be reduced to a simple standard form in the neighborhood of an analytic singular point, i. e., a point at which $X(x, y)$ and $Y(x, y)$ are analytic, but vanish simultaneously. Although the method fails to be directly applicable to certain special cases, it has shown itself to be of sufficient

* Presented to the Society September 9, 1926.

† Journal de l'Ecole Polytechnique, vol. 21, p. 161. See also Picard, Traité d'Analyse, Paris, Gauthier-Villars, 1908, vol. 3, p. 34. 